\title{
Isolated Left Insular Infarction and Acute Psychosis: A Misleading Clinical Presentation
}

\author{
Komi Assogba ${ }^{1 *}$, Michel F Kayem Tassa ${ }^{1}$, Kossivi Martin Apetse $^{1}$, Damsane Lamboni ${ }^{2}$, Tajeuna JJ \\ Dongmo $^{1}$, Joshue E Diatewa ${ }^{1}$, Komi Agbotsou ${ }^{1}$, Vinyo Codja ${ }^{1}$, Faiza M Mboumba ${ }^{1}$, Abdullah Blakime ${ }^{1}$ and \\ Koffi AA Balogou ${ }^{1}$
}

${ }^{1}$ Department of Neurology, Togo

${ }^{2}$ Department of Thoracic surgery, Togo

*Corresponding author: Komi Assogba, Professor of Neurology, Campus Teaching Hospital of Lomé, Togo.

Received Date: November 21, 2018

Published Date: December 10, 2018

\begin{abstract}
Introduction: The insular cortex is a hidden structure located in the Sylvian fissure surrounded by the temporal, frontal, and parietal lobes. It is a complex structure interconnecting various brain regions. The insular lobe plays multiple roles including visceromotor, viscerosensory and limbic functions. These roles contribute to control autonomic, memory, behavior and language system. The arteries supplying the insular area are mainly originate from the M2 segment of the middle cerebral artery. The isolated insular infarction stroke is extremely rare. The objective was to report this case study with literature reviewed.

Clinical study: The 60-year-old patient was admitted to our tertiary hospital with behavioral complains symptoms. He had a pass history of essential thrombocythemia under hydroxyurea. The disorders were started suddenly and marked by behavioral disturbances such as self runaways, aggression and a feeling of strangulation. The clinical examination revealed personality disorders with delusions of persecution type, auditory hallucinations, unmotivated laughing and crying, and speech disorders such as tachylalia and paraphasia. There was an unpleasant sensation of the left hemi-body and no motor deficit or cranial nerves involvement. Brain magnetic resonance imaging revealed isolated infarction of the left insular cortex (Figure 1). The diagnostic of left insular infarction with psychiatric manifestations was made and the patient underwent anti platelet treatment and remote psychiatric control drugs. After three months of follow up, the patient had got good recovery of language, mood and behavioral disorders.
\end{abstract}

Conclusion: The polymorphism of insular stroke clinical presentation raises the major interest of after stroke clinical and neuropsychological follow up to improve the quality of life of patient. The prognosis of the isolated insular infarction is often favorable but under appropriate diagnostic.

Keywords: Acute psychosis; Insular infarction; Clinical symptoms; MRI findings; Stroke

\section{Introduction}

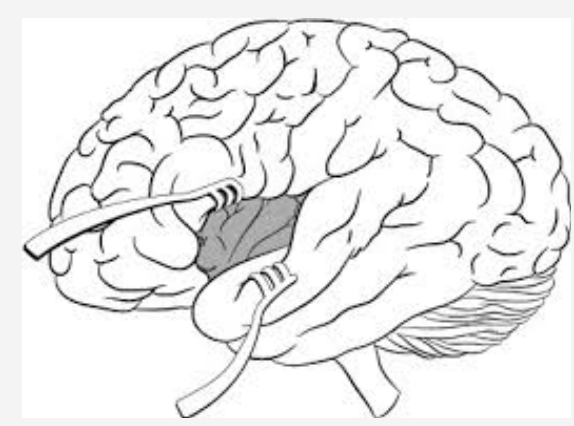

Figure 1: Frontal section of the brain showing the insular cortex.
The insular cortex is a hidden structure located in the Sylvian fissure surrounded by the temporal, frontal, and parietal lobes (Figure1). It is a complex structure interconnecting various brain regions [1-3]. The insular lobe plays multiple roles including visceromotor, viscerosensory and limbic functions. These roles contribute to control autonomic, gustatory, auditory, volitional swallowing, and speech and language function as well as somatosensory perception and emotional response [4-6]. The insula may be implicated in the coordination between internal and external information through emotional subjective awareness and facilitate motor recovery in stroke [7-9]. The arteries supplying the insular area mainly originate from the M2 segment of the middle 
cerebral artery [10-12]. The objective was to present a case of isolated left insular infarction and to discuss the real impact of the insular cortex on neuropsychiatric disorders in light with the literature.

\section{Clinical Presentation}

The patient was admitted to our tertiary hospital with behavioral complains. This 60-year-old patient, ambidexter hand used with a history of essential thrombocythemia under hydroxyurea, was admitted 4 hours after the onset of the symptoms. No antecedent of psychiatric disorders. The disorders were started suddenly and marked by behavioral disturbances such as self runaways, aggression and a feeling of strangulation. The clinical examination revealed personality disorders with delusions of persecution type, auditory hallucinations, unmotivated laughing and crying, and speech disorders such as tachylalia and paraphasia. There was no fever, acute nausea and vomiting like but complained with an unpleasant sensation of the left hemi-body.
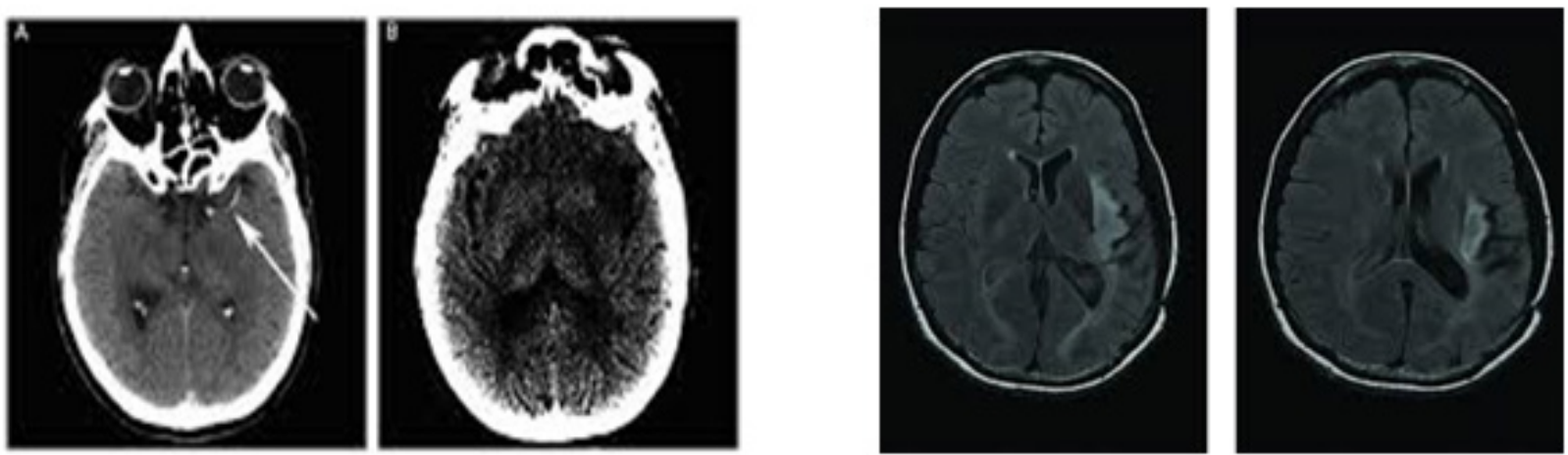

Figure 2: Computered axial tomography scan showing the left insular infarction with and without contrast.
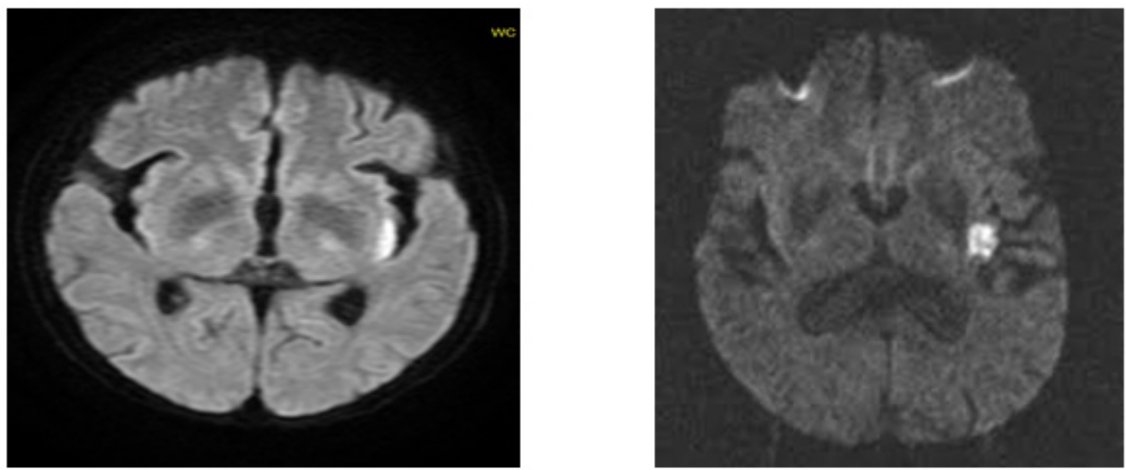

Figure 3: Brain MRI diffusion showing high signal T2 weighted of insular cortex infarction.

There were no motor and sensory deficits or cranial nerves involvement. It was difficult to test the taste and the eye field. The first clinical diagnostic was acute psychosis. Blood sample biological tests including electrolytes control and CSF analyses were performed and become normal. Brain CT scan and magnetic resonance imaging revealed isolated infarction of the left insula cortex (Figure 2 \& 3). The diagnostic of left insular infarction with psychiatric manifestations was made and the patient underwent anti platelet treatment. He was also put under remote psychiatric control drugs. He was also following up for psychological support, speech therapy and vascular risk factors control. After three months of follow up, the patient had got good recovery of mood and behavioral disorders. It is unusual presentation of insular infarction and lead to discuss the real functions of the insular cortex and the impact of laterality on neuropsychiatric disorders.

\section{Discussion}

The main limitation of our study relies on a non-systematic and incomplete evaluation of clinical and neuropsychological deficits at initial admission. It can exist misdiagnosis lesions of insula cortex due to our limited research technical platform tools and therefore other bias must come from the recruitment of the patients due to admission difficulties or cultural mindset. As many people look like mad man are run alongside public road every day may benefit of brain imaging with appropriate care. This observational case study raises the evidence based of implication of insular cortex in the emotional behavior, motor and sensory perception, and the language. These symptoms are rare in right hand patients with left insular lesion. To our knowledge, this clinical presentation is unique in our daily practice because the isolated insular infarct lesions is very rare.

The behavioral and mood disturbances may evocate a priori the orbito-frontal or prefrontal lesion, and the sensory impairment symptoms the postero lateral thalamic or parietal lesions. The symptoms can also lead to acute psychosis and raise the real need of performing brain scan in psychiatric patient. For instance, the association between the insular cortex lesions and autonomic 
control has been elucidated by several previous authors $[2,5,7]$. Isolated left insular infarction is a rare condition. In one study, among patient with first episode of ischemic stroke, only $0.08 \%$ had isolated insular infarction [13]. The findings of multidomain cognitive impairment in this patient suggest that the insular cortex is a convergent structure; thus, the deficits caused are more likely to be the consequence of diaschisis rather than damage of a specialized or isolated center itself.

Due to the complexity of the behavioral process, it is challenging to draw conclusions about the role of the insular cortex in speech and mood production. Left isolated insular infarction (III) was previously reported to cause these disorders $[6,9,14]$. The insular cortex is activated in the phonological process, executive function and behavioral control. Therefore, III may cause a disconnection between the primary and supplementary motor cortex, which may be a result of neuronal network disruption between the anterior insula and frontal lobe and/or cingulate regions, leading to executive dysfunction of motor speech and personality balance control $[4,15]$.

The insular cortex is associated with gustatory, visual, and verbal memory. Patients with a left insular infarction have more impairment in verbal and logical memory than patients with a right insular stroke. The disruption of the connection between the left temporal lobe and the left insular cortex may play a role in this deficit $[14,16]$. Since the posterior insular cortex is adjacent to the semantic storage areas, this infarction may cause network disconnection between these areas, resulting in difficulty retrieving previously learned knowledge. Even though various types of aphasia were reported in strokes involving the insular cortex, isolated insular infarction with tachylalia and paraphasia is very uncommon $[11,17]$.

Anatomically, the insular lobe is in the central part of the structures that are important in language functions, including the frontal operculum and Wernicke's area. The disconnection of these structures and insular area may contribute to language dysfunction after insular damage $[1,3,18]$. Our clinical observation report has showed that III can result in a vast array of deficits. The most frequent are being somatosensory, speech, autonomic, and motor symptoms. These deficits are in line with known functions of the insula, based on previous electrophysiological, functional and cytological studies $[1,17,19]$. While a combination of somatosensory, language and motor disturbances may suggest a larger MCA stroke, certain unusual concomitant symptoms should raise suspicion of a smaller stroke in the multimodal insular areas. Furthermore, although IIS may sometimes result in proportional motor or sensory deficits, mimicking a lacunar infarct, but in our case, there were no motor sensory and cranial nerves impairment. Less commonly, III can also present as a posterior circulation stroke, with acute vertigo, nausea and/or ataxia $[5,6,20]$.

Another presentation of IIS is made with behavioral and mood disturbances, delusion and aggression that can mimic acute psychosis or frontal lobe involvement, as found with our reported patient. The functional prognosis of III appears to be excellent as our patient was asymptomatic with good recovery of psychiatric symptoms after 3 months of follow up. This suggests that surrounding or distant structures can generally compensate limited insular damage $[9,10,21]$. A broad range of evidence demonstrates that reorganization of forebrain structures after a stroke is much more likely to cause recovery of function in childhood than further loss of function in adults $[1,9,22]$. The author in the previous study had determined the predictive risk factor of re-occlusion of MCA in the case of insula infarction by using insular score, which is simple, practical, and highly reproducible visual assessment than NIHSS score. It should be noted that these findings were applied to patients who present with small infarcts $[2,15,23]$.

\section{Conclusion}

The polymorphism of insula stroke clinical presentation raises the major interest of after stroke clinical and neuropsychological follow up to improve the quality of life of patient. The prognosis of the isolated insular infarction is favorable. In a case series, more than half of patients with isolated insular infarction or minimal extension to adjacent areas completely.

\section{Acknowledgement}

None.

\section{Conflict of Interest}

No conflict of interest.

\section{References}

1. Stephani C, Fernandez-Baca VG, Maciunas R, Koubeissi M, Lüders HO (2011) Functional neuroanatomy of the insular lobe. Brain Struct Funct 216(2): 137-149.

2. Craig AD (2014) Topographically Organized Projection to Posterior Insular Cortex from the Posterior Portion of the Ventral Medial Nucleus (VMpo) in the Long-tailed Macaque Monkey. J Comp Neurol 522(1): 3643.

3. Penfield W, Faulk ME (1955) The insula; further observations on its function. Brain 78(4): 445-470.

4. Julayanont P, Doungporn Ruthirago, De Toledo JC (2016) Isolated left posterior insular infarction and convergent roles in verbal fluency, language, memory, and executive function. Proc (Bayl Univ Med Cent 29(3): 295-297.

5. Lemieux F, Lanthier S, Chevrier MC, Gioia L, Rouleau I, et al. (2012) Insular Ischemic Stroke: Clinical Presentation and Outcome. Cerebrovasc Dis Extra 2: 80-87.

6. Ibanez A, Gleichgerrcht E, Manes F (2010) Clinical effects of insular damage in humans. Brain Struct Funct 214(5-6): 397-410.

7. Markostamou I, Rudolf J, Tsiptsios I, Kosmidis MH (2015) Impaired executive functioning after left anterior insular stroke: a case report. Neurocase 21(2): 148-153.

8. Cereda C, Ghika J, Maeder P, Bogousslavsky J (2002) Strokes restricted to the insular cortex. Neurology 59(12): 1950-1955.

9. O'Brien LD, Sticht MA, Mitchnick KA, Limebeer CL, Parker LA, et al. (2014) CB1 receptor antagonism in the granular insular cortex or somatosensory area facilitates consolidation of object recognition memory. Neurosci Lett 578: 192-196.

10. Borowsky R, Cummine J, Owen WJ, Friesen CK, Shih F, et al. (2006) FMRI of ventral and dorsal processing streams in basic reading processes: insular sensitivity to phonology. Brain Topogr 18 (4): 233- 239.

11. Ture U, Yașargil MG, Al-Mefty O, Yașargil DC (2000) Arteries of the insula. J Neurosurg 92 (4): 676-687.

12. Kurth F, Eickhoff SB, Schleicher A, Hoemke L, Zilles K, et al. (2010) Cytoarchitecture and probabilistic maps of the human posterior insular cortex. Cereb Cortex 20(6): 1448-1461. 
13. Guggisberg AG, Mathis J, Schnider A, Hess CW (2011) Why do we yawn? The importance of evidence for specific yawn-induced effects. Neurosci Biobehav Rev 35(5): 1302-1304.

14. Krestel H, Weisstanner Ch, Hess ChW, Bassetti CL, Nirkko A, et al. (2015) Insular and caudate lesions release abnormal yawning in stroke patients. Brain Struct Funct 220(2): 803-812.

15. Baier B, zu Eulenburg P, Glassl O, Dieterich M (2011) Lesions to the posterior insular cortex cause dysarthria. Eur J Neurol18(12): 14291431.

16. Suto T, Meguro K, Nakatsuka M, Kato Y, Tezuka K, et al. (2014) Disorders of "taste cognition" are associated with insular involvement in patients with Alzheimer's disease and vascular dementia: "memory of food is impaired in dementia and responsible for poor diet." Int Psychogeriatr 26 (7): 1127-1138.

17. Souza LC, Yoo AJ, Chaudhry ZA, Payabvash S, Kemmling A, et al. (2012) Malignant CTA collateral profile is highly specific for large admission DWI infarct score and poor outcome in acute stroke. AJNR Am J Neuroradiol 33: 1331-1336.
18. Baier B, Conrad J, Zu Eulenburg P, Best C, Müller-Forell W, et al. (2013) Insular strokes cause no vestibular deficits. Stroke 44(9): 2604-2606.

19. Liou LM, Guo YC, Lai CL, Tsai CL, Khor GT (2010) Isolated pure ataxia after left insular cortex infarction. Neurol Sci 31(1): 89-92.

20. Craig AD (2011) Significance of the insula for the evolution of human awareness of feelings from the body. Ann N Y Acad Sci 1225: 72-82.

21. Veldhuijzen DS, Greenspan JD, Kim JH, Lenz FA (2010) Altered pain and thermal sensation in subjects with isolated parietal and insular cortical lesions. Eur J Pain 14(5): 535-541.

22. Kamalian Sh, Kemmling A, Borgie RC, Morais LT, Payabvash S, et al. (2013) Admission Insular Infarction $>25 \%$ is the Strongest Predictor of Large Mismatch Loss in Proximal MCA Stroke. Stroke 44(11): 30843089 .

23. Carota A, Annoni JM, Marangolo P (2007) Repeating through the insula: evidence from two consecutive strokes. Neuroreport 18(13): 1367 1370. 